\title{
MULTIBODY MODELLING OF THE VERTICAL APPARENT MASS AND FORE-AND-AFT CROSS-AXIS APPARENT MASS OF THE SEATED HUMAN BODY WITH A BACKREST
}

\author{
Guangtai Zheng, Yi Qiu and Michael J Griffin \\ Human Factors Research Unit, Institute of Sound and Vibration Research, \\ University of Southampton, United Kingdom
}

\section{Introduction}

Backrests affect the biodynamic responses of the body to whole-body vibration. ${ }^{1-2}$ Models of the seated human body exposed to whole-body vibration should therefore take into account the effects of backrests. The purpose of this study was to identify the characteristics of a model needed to reflect both the vertical in-line apparent mass and the fore-and-aft cross-axis apparent mass measured at both the seat and backrest and consider the contribution of each body segment to the dynamic response.

\section{Methods}

The human body was represented by five rigid bodies: upper-body, middle-body, pelvis, thighs, and legs (Figure 1). The rigid bodies were inter-connected by pin joints with rotational stiffness and damping. The normal and shear deformation of the pelvis, thigh and back tissues at the seat-occupant interfaces were simulated using linear springs and dampers. The equations of motion of the model were derived using the Lagrange formulation. The geometric and inertial properties were derived from the literature. ${ }^{3}$ The mechanical properties were obtained by minimizing the error functions between the computed and measured moduli of the median apparent masses over the frequency range 0.3 to $20 \mathrm{~Hz}$.

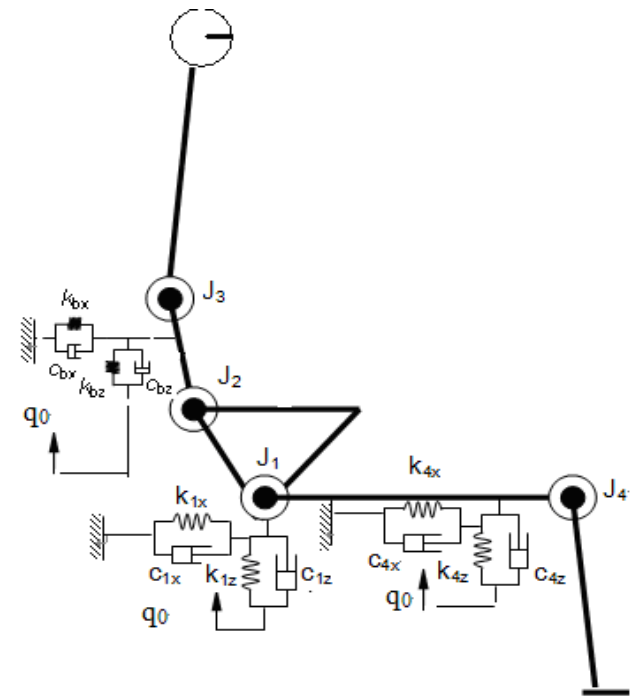

(i)

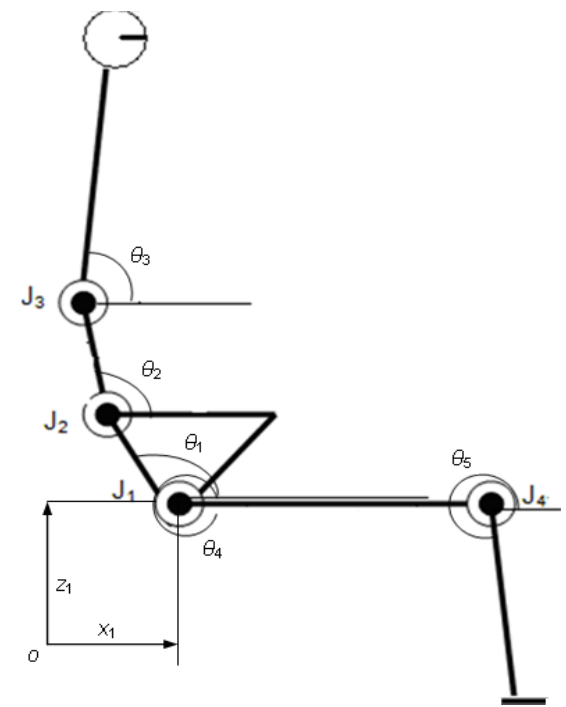

(ii)

Figure 1 A two-dimensional multi-body biomechanical model in the normal upright posture: (i) model structure; (ii) motion description. 


\section{Results}

The predicted moduli and phases of the vertical apparent mass and fore-and-aft apparent mass at the seat and back are compared with experimental data in Figure 2.,

(i) Modulus

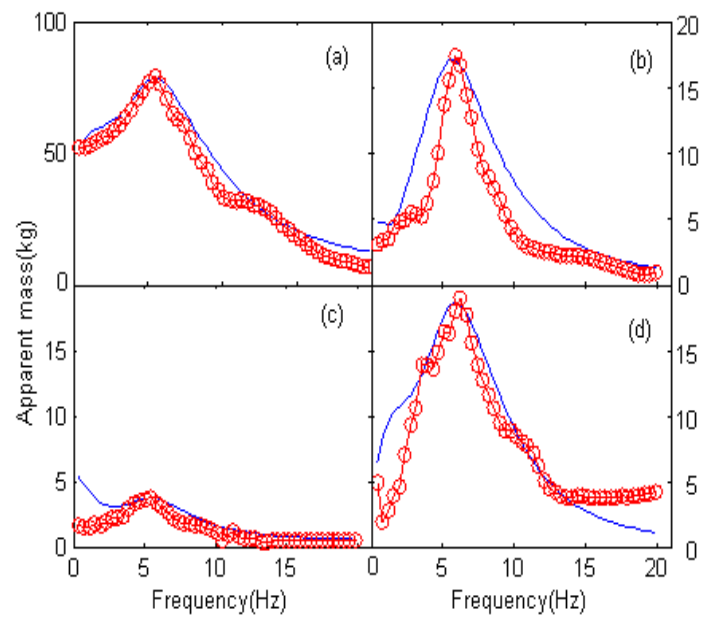

(ii) Phase

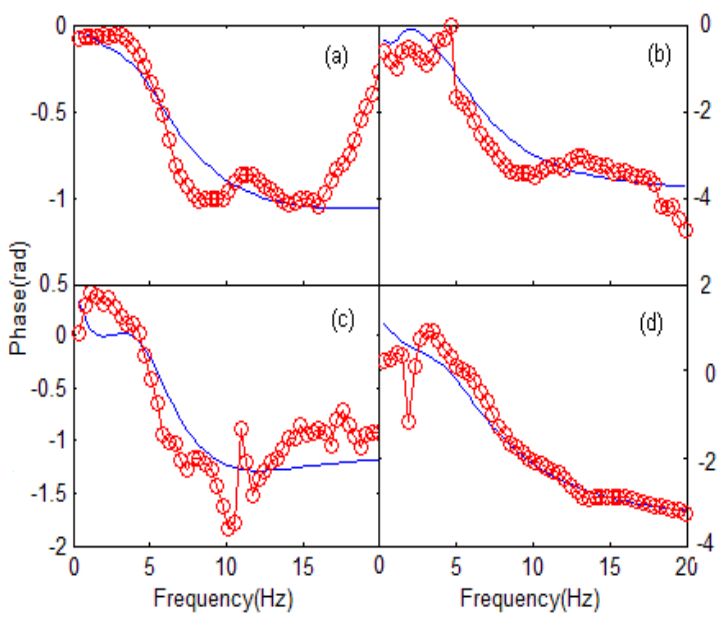

Figure 2 Predicted and measured vertical and fore-and-aft cross-axis apparent mass of the human body with backrest: (i) modulus, (ii) phase. contact point at the upper lumbar spine); - - - measured ${ }^{2,4}$; (a) vertical apparent mass on the seat; (b) fore-and-aft cross-axis apparent mass on the seat; (c) vertical apparent mass on the back; (d) fore-and-aft cross-axis apparent mass on the back.

\section{Discussion}

The model is capable of predicting apparent masses in reasonable agreement with the measured data. A parameter sensitivity analysis indicated that the apparent masses at the seat and back in the vertical and fore-and-aft directions were mainly influenced by the vertical pelvis stiffness and the rotational motion of the lumbar spine and pelvis.

Contact with a backrest affects the apparent mass and so backrest contact should be taken into account in biodynamic models of human responses to vibration.

\section{References}

1. Fairley, T.E. and Griffin, M.J. (1989). The apparent mass of the seated human body: vertical vibration. Journal of Biomechanics, 22, 81-94.

2. Nawayseh, N. and Griffin, M.J. (2003). Non-linear dual-axis biodynamic response to vertical wholebody vibration. Journal of Sound and Vibration, 268, 503-523.

3. National Aeronautical and Space Administration (1978). NASA Reference Publication 1024. Anthropometric source book, volume1: anthropometry for designers.

4. Qiu, Y. and Griffin, M.J. (2009). Biodynamic responses of the seated human body to single-axis and dual-axis vibration, Proceedings of $4^{\text {th }}$ International Conference on Whole-Body Vibration Injuries, June 2 to 4, 2009, Montreal, Canada. 